\title{
Impact of a genomic classifier of metastatic risk on postoperative treatment recommendations for prostate cancer patients: a report from the DECIDE study group
}

\author{
Ketan Badani ${ }^{1}$, Darby J. S. Thompson ${ }^{2}$, Christine Buerki ${ }^{3}$, Elai Davicioni ${ }^{3}$, Jill \\ Garrison ${ }^{4}$, Mercedeh Ghadessi ${ }^{3}$, Anirban P. Mitra ${ }^{5}$, Penelope J. Wood ${ }^{3}$, John \\ Hornberger ${ }^{4,6}$ \\ ${ }^{1}$ Department of Urology, Columbia University, New York, NY USA \\ 2 EMMES Canada, Burnaby, BC CAN \\ ${ }^{3}$ GenomeDx Biosciences Inc., Vancouver, BC CAN \\ ${ }^{4}$ Cedar Associates LLC, Menlo Park, CA USA \\ ${ }^{5}$ University of Southern California, Los Angeles, CA USA \\ ${ }^{6}$ Stanford University, Stanford, CA USA \\ Correspondence to: Ketan Badani, email: kb2388@mail.cumc.columbia.edu \\ Keywords: prostate cancer, prognosis, metastasis, decision impact, patient management, clinical practice \\ Received: March 5, $2013 \quad$ Accepted: April 7, $2013 \quad$ Published: April 8, 2013
}

This is an open-access article distributed under the terms of the Creative Commons Attribution License, which permits unrestricted use, distribution, and reproduction in any medium, provided the original author and source are credited.

ABSTRACT:

Background: Only a minority of prostate cancer patients with adverse pathology and biochemical recurrence (BCR) post radical prostatectomy (RP) experience metastasis and die from prostate cancer. Improved risk prediction models using genomic information may enable clinicians to better weigh the risk of metastasis and the morbidity and costs of treatment in a clinically heterogeneous population.

Purpose: We present a clinical utility study that evaluates the influence on urologist treatment recommendations for patients at risk of metastasis using a genomic-based prediction model (Decipher ${ }^{\mathrm{TM}}$ ).

Methods: A prospective, pre-post design was used to assess urologist treatment recommendations following RP in both the adjuvant (without any evidence of PSA rise) and salvage (BCR) settings. Urologists were presented de-identified pathology reports and genomic classifier (GC) test results for 24 patients from a previously conducted GC validation study in high-risk post-RP men. Participants were fellowship trained, high-volume urologic oncologists $(n=21)$ from 18 US institutions. Treatment recommendations for secondary therapy were made based solely on clinical information (pre-GC) and then with genomic biomarker information (post-GC). This study was approved by an independent IRB.

Results: Treatment recommendations changed from pre-GC to post-GC in $43 \%$ of adjuvant, and in $53 \%$ of salvage setting case evaluations. In the adjuvant setting, urologists changed their treatment recommendations from treatment (i.e. radiation and/or hormones) to close observation post-GC in $27 \%$ of cases. For cases with low GC risk ( $<3 \%$ risk of metastasis), observation was recommended for $79 \%$ of the case evaluations post-GC. Consistent trends were observed in the salvage setting.

Conclusion: These results indicate that urologists across a range of practice settings are likely to change treatment decisions when presented with genomic biomarker information following RP. Implementation of genomic risk stratification into routine clinical practice may better direct treatment decision-making post-RP. 


\section{INTRODUCTION}

Prostate cancer presents a significant population health burden in the United States. As the most frequently diagnosed cancer among men, almost 240,000 new cases are projected for 2013 [1]. About half of these men will be treated with radical prostatectomy (RP) [2] and while many will achieve a durable cure, up to $50 \%$ will present with one or more adverse pathology features such as, seminal vesicle invasion (SVI), extracapsular extension (ECE) or positive surgical margins $[3,4]$. Although these patients are considered by guidelines to be at an increased risk for disease progression, only a minority will develop metastatic disease and ultimately die of prostate cancer [5]. Further, while close monitoring with postoperative PSA testing can identify men at risk, the time to biochemical recurrence (BCR) after RP is not predictive for metastatic disease [6]. And, while PSA doubling time (PSAdt) is a good surrogate for aggressive disease, its accurate determination may not be possible in all patients as it requires a certain timeframe during which clinical progression may occur [7].

Treatment recommendations from the National Comprehensive Cancer Network (NCCN) guidelines post-RP include radiation and/or hormone therapy or observation (active surveillance). These guidelines are based in part on results from three independent phase III randomized clinical trials that have demonstrated improvements in BCR-free, metastasis-free and cancerspecific survival in high-risk post-RP men treated with radiation therapy [8-10]. Despite this, deciding on appropriate use of radiation therapy post-RP remains a challenging task. Inappropriate or over-utilization of secondary therapy in this population is of great concern because of the knowledge that most clinically highrisk, post-RP patients will never develop metastasis. Recognizing these factors, guidelines state that "predicting prognosis is essential for patient decisionmaking, treatment selection, and adjuvant therapy"[4]. To better guide treatment decisions, a need persists to more accurately characterize a patient's risk of metastasis.

Assessment of risk when considering postoperative secondary therapy is currently conducted based on individual clinicopathologic (clinical) variables and/or through use of nomograms [11]. However, the ability of these clinical variables to identify patients at substantially higher risk of metastasis and lethal prostate cancer is limited. Genomic features in the primary tumor reflect the true biological potential for disease progression and metastasis. Novel risk prediction tools that use such features can therefore provide the direct measure of risk that is needed. One such tool is a postoperative genomic classifier (GC) test (Decipher ${ }^{\mathrm{TM}}$, GenomeDx Biosciences, San Diego, CA) that uses a whole-transcriptome microarray assay for analysis of gene activity in formalinfixed paraffin embedded prostate cancer specimens.

\begin{tabular}{|l|l|l|l|}
\hline \multicolumn{1}{|l}{$\begin{array}{l}\text { Table 1: Characteristics of } \\
\text { participating in study. }\end{array}$} & urologists \\
\hline & Total & $\begin{array}{l}\text { Adjuvant } \\
\text { Evaluation }\end{array}$ & $\begin{array}{l}\text { Salvage } \\
\text { Evaluation }\end{array}$ \\
\hline & $\mathrm{n}=21$ & $\mathrm{n}=20$ & $\mathrm{n}=15$ \\
\hline & No. (\%) & No. (\%) & No. (\%) \\
\hline Practice Setting & & & \\
\hline Tertiary Care & $13(62 \%)$ & $12(60 \%)$ & $9(60 \%)$ \\
\hline $\begin{array}{l}\text { Community } \\
\text { (hospital or private) }\end{array}$ & $8(38 \%)$ & $8(40 \%)$ & $6(40 \%)$ \\
\hline $\begin{array}{l}\text { Number of years in } \\
\text { practice }\end{array}$ & 8.1 & 8.3 & 7.8 \\
\hline Mean & $3-25$ & $3-25$ & $3-25$ \\
\hline Range & & & \\
\hline $\begin{array}{l}\text { Number of Radical } \\
\text { Prostatectomy per } \\
\text { year }\end{array}$ & 184 & 179 & 200 \\
\hline Mean & $30-300$ & $30-300$ & $30-300$ \\
\hline Range & & & \\
\hline Geographic Region & & & \\
\hline West/South Central & $4(20 \%)$ & $4(20 \%)$ & $3(20 \%)$ \\
\hline South East & $4(20 \%)$ & $4(20 \%)$ & $3(20 \%)$ \\
\hline Mid Atlantic & $4(20 \%)$ & $3(15 \%)$ & $2(13 \%)$ \\
\hline North East & $5(25 \%)$ & $5(25 \%)$ & $5(33 \%)$ \\
\hline North Central & $4(20 \%)$ & $4(20 \%)$ & $2(13 \%)$ \\
\hline
\end{tabular}

Developed in collaboration with the Mayo Clinic, this GC was designed to predict early clinical metastasis following RP [12]. In a blinded clinical validation study of a contemporary high-risk population of post-RP men with adverse pathology, the GC test was found to more accurately predict metastasis post-RP than individual clinical variables, combinations of clinical variables or currently used nomograms $[13,14]$.

In assessing a novel molecular test, experts have recommended that evidence be collected not only on the clinical validity of the test, but also on how use of the test influences clinical practice management, a wellestablished measure of the test's clinical utility $[15,16]$. The primary objective of this study was to determine how urologists' knowledge of the GC test results influenced adjuvant and salvage treatment recommendations following RP.

\section{RESULTS}

Participating physicians were all practicing, high surgical volume urologists performing an average of 184 RPs per year (Table 1). Twenty-one urologists from 18 different institutions across the US participated: 20 in the adjuvant, and 15 in the salvage settings. Fourteen of these 


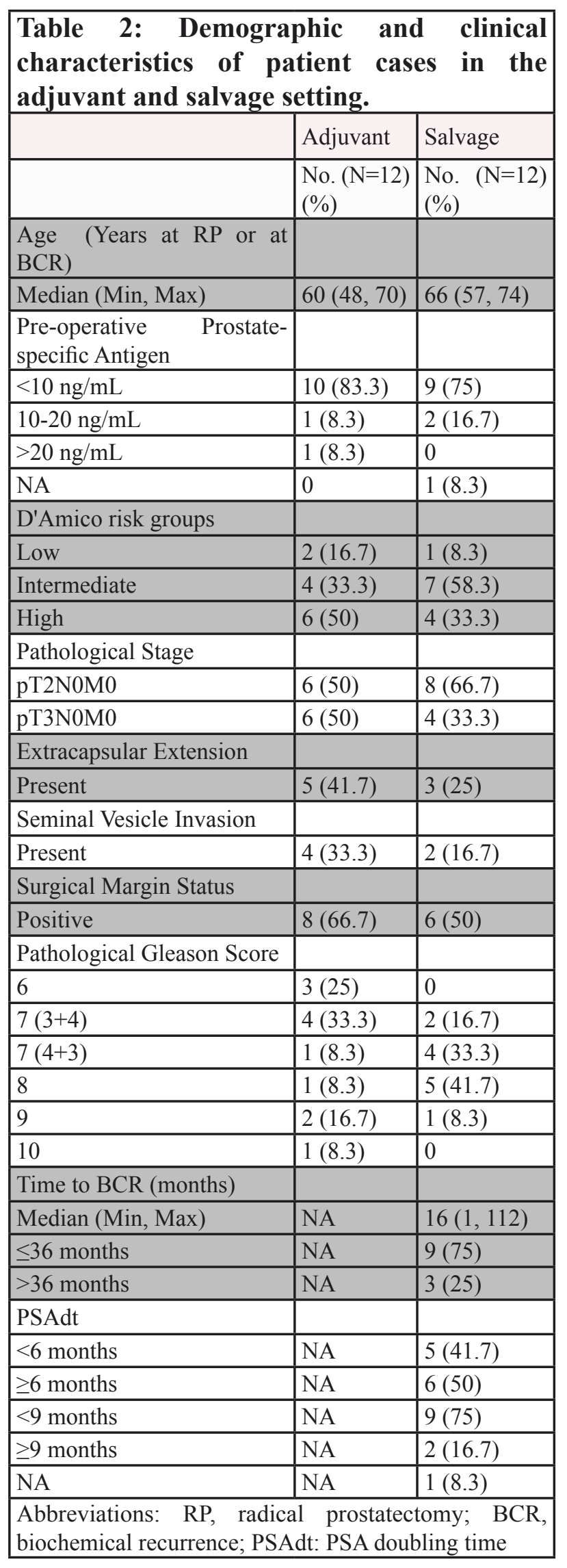

urologists completed assessment of cases in both substudies. Of the 21 urologists, $38 \%(\mathrm{n}=8)$ practice in a community-based hospital or private practice setting and $62 \%(\mathrm{n}=13)$ practice in tertiary care centers, the majority $(85 \%)$ of which are National Cancer Institute (NCI) designated comprehensive cancer centers. Urologists had been practicing and performing surgery for 3 to 25 years (mean 8.1 years) and all have extensive experience managing and treating patients with prostate cancer both before and after RP.

Characteristics of the twelve patients in each of the adjuvant and salvage settings are provided in Table 2. Half of the adjuvant patient cases were pre-operatively deemed low to intermediate risk according to D'Amico risk groups but were all subsequently up-graded/staged postoperatively. Furthermore, $75 \%$ of these cases presented with a pathologic Gleason score $\geq 7$, and $36 \%$ were $\geq 65$ years of age at the time of surgery. For cases reviewed in the salvage setting, $75 \%$ had a time to BCR $\leq 36$ months, and $75 \%$ presented with a rapid PSAdt $(<9$ months). The majority (58\%) of these cases were $\geq 65$ years of age at the time of BCR.

In the adjuvant treatment setting, $43 \%(95 \% \mathrm{CI}$ : $37-49 \%$ ) of recommendations changed following review of the GC test results (Table 3). Specifically, among case evaluations with a pre-GC recommendation involving treatment, $27 \%$ (95\% CI: $19-35 \%)$ of recommendations changed to observation post-GC. Notably, for case evaluations with a pre-GC recommendation of radiation alone $(\mathrm{n}=100), \quad 31 \% \quad(95 \% \quad \mathrm{CI}: \quad 22-41 \%)$ changed to observation post-GC (Table 3). Among the case evaluations where observation was initially chosen $(n=114)$, treatment was recommended for $37 \%$ of case evaluations post-GC, primarily in favor of radiation therapy alone (37/42). This can be visualized in Figure 1 , which shows how in comparison to pre-GC, post-GC urologists' recommendations for observation or treatment (radiation and/or hormones) aligned to a high degree with the risk assigned by the GC test. Detailed results for all possible combinations of pre- and post-GC treatment recommendations are provided in Supplementary Table S1.

In the salvage setting, treatment recommendations changed 53\% (95\% CI: $45-60 \%)$ of the time (Table 3). Among case evaluations with a pre-GC recommendation involving treatment $(\mathrm{n}=143), 16 \%(95 \%$ CI: $11-23 \%)$ changed to observation post-GC. Expectedly, there were fewer pre-GC recommendations of observation $(n=31)$ for case evaluations with BCR. Of these, $61 \%(n=19)$ were recommended to change from observation to involve treatment post-GC with radiation alone $(\mathrm{n}=12)$ or in combination with hormonal therapy $(n=7)$ (Table 3). Similar to the analysis of the adjuvant setting above, we observe a trend that shows alignment of observation versus treatment recommendations with the risk assigned by the GC test, even though treatment recommendation 


\begin{tabular}{l}
\hline $\begin{array}{l}\text { Table 3: Effect of the GC test result on urologists treatment recommendations post radical } \\
\text { prostatectomy. }\end{array}$ \\
\hline Treatment Recommendation
\end{tabular}

rates were higher overall in the salvage setting (Figure S1).

Results were virtually unchanged when the possible correlation of recommendations from the same urologist (i.e. intra-observer correlation) were accounted for (refer to Supplementary Material).

To further examine the impact of the relationships between clinical variables and the GC test results on urologists' treatment recommendations, we evaluated the proportion of urologists recommending treatment preand post-GC over the complete set of case evaluations, as well as within individual clinical variables for high and low GC risk patients (Table S2). GC risk was established based on whether the predicted probability of developing metastasis was above (high GC risk) or below (low GC risk) the average risk for the original study population (see methods). Overall, in the adjuvant setting, treatment was recommended $52 \%$ of the time pre-GC. Post-GC, those with a low GC risk were recommended treatment only $21 \%$ of the time (i.e., $79 \%$ recommended to observation) compared to those with a high GC risk who were recommended treatment $90 \%$ of the time $(\mathrm{p}<0.0001)$. Similarly, in the salvage setting, the overall proportion of treatment recommendation was $79 \%$ pre-GC, but post-GC fell to $75 \%$ in the low GC risk group and rose to $85 \%$ in the high-risk GC group $(\mathrm{p}=0.031)$.
When evaluating individual clinical variables in the adjuvant setting (Table S2), cases with ECE present had the highest marginal rate of treatment recommendation pre-GC (77\%); this fell to $28 \%$ for low GC risk case evaluations and rose to $97 \%$ for high GC risk case evaluations post-GC $(\mathrm{p}<0.0001)$ (Figure 2). Similarly, in cases with positive surgical margins, $54 \%$ were recommended treatment pre-GC. Treatment recommendation dropped to $18 \%$ for cases with low GC risk and rose to $93 \%$ in high $\mathrm{GC}$ risk cases $(\mathrm{p}<0.0001)$. For cases with pathological Gleason score $\geq 7$ disease, $65 \%$ were recommended treatment pre-GC; among those with low GC risk only $25 \%$ were recommended treatment versus $90 \%$ of those cases with high GC risk $(\mathrm{p}<0.01)$. The largest magnitude in change was observed in cases with SVI. Pre-GC, $70 \%$ of SVI cases were recommended treatment, but among those cases with low GC risk, only $23 \%$ were recommended treatment in the presence of SVI. In high GC risk cases with SVI, 95\% were recommended for treatment $(p<0.0001)$. These results reinforce the impact of the GC test and indicate that the rate of treatment may be strongly associated with the GC risk (or probability of developing metastasis) than any other clinical variable (Table S2, Figure 2).

In the salvage setting, pre-GC the main drivers for recommendation of treatment were PSAdt and time 
to BCR. Cases with a rapid PSAdt of $<6$ months were recommended for treatment by $93 \%$ of urologists preGC. However, the proportion dropped to $73 \%$ within low GC risk patients, post-GC. For cases with longer PSAdt (and hence a presumed better prognosis), only 14 (47\%) recommendations for treatment were made pre-GC, but this increased to $25(83 \%)$, post-GC, and all of these cases had high GC risk. As in the adjuvant setting, presence or absence of individual clinical variables (except for margin status) did not influence recommendations to treat post$\mathrm{GC}$; in this setting, GC risk was the main driver (Table $\mathrm{S} 2$, Figure S2).

Results for univariable and multivariable regression models for recommendation of treatment pre- and post-GC are included in Table S3. In the adjuvant setting, clinical variables influence urologists' recommendation to treat pre-GC. When the urologists are aware of the genetic risk, post-GC, only GC risk $(\mathrm{p}<0.0001)$ and $\mathrm{ECE}(\mathrm{p}=$ 0.018 ) have a significant impact on the recommendation for treatment. While not significant in the salvage setting, GC risk has the lowest p-value of all variables in the postGC multivariable model. Since cases were not randomly sampled from the general population, these results are considered exploratory.

To measure recommended changes in treatment intensity, we established a baseline clinical perception of risk (hereafter referred to as perceived risk). Cases were considered low perceived risk if less than half of urologists recommended treatment and high perceived risk if more than half recommended treatment in the absence of the $\mathrm{GC}$ test results. In the adjuvant and salvage settings we observed that if perceived risk was high but GC risk was low, then, respectively, $50 \%$ and $46 \%$ of recommendations reduced treatment intensity post-GC (e.g., radiation to observation or radiation/hormone combination to radiation only) (Table 4). Very few recommendations were made that increased treatment intensity, (only 5\% and $3.8 \%$, respectively for adjuvant and salvage treatment recommendations). Conversely, for cases with an initial low perceived risk but high GC risk, we observed a $55 \%$ and $58 \%$ increase in treatment intensity in both the adjuvant and salvage settings, respectively. Influence of $\mathrm{GC}$ risk on change in intensity for all clinical risk categories and treatment settings were highly statistically significant $(\mathrm{p}<0.0001)$. Furthermore, a multivariable model adjusting for the pre-GC clinical risk showed that $\mathrm{GC}$ risk influenced change in treatment recommendation intensity $(\mathrm{p}<0.0001)$.

To understand the extent to which the GC test result impacts confidence in making a treatment recommendation, urologists were asked to report on the degree to which they felt confident in the treatment recommendation made for case evaluations both pre- and post-GC, as well as, the extent to which they felt the GC test result influenced those treatment recommendations. Results showed that for case evaluations where a treatment

\section{Pre-GC}

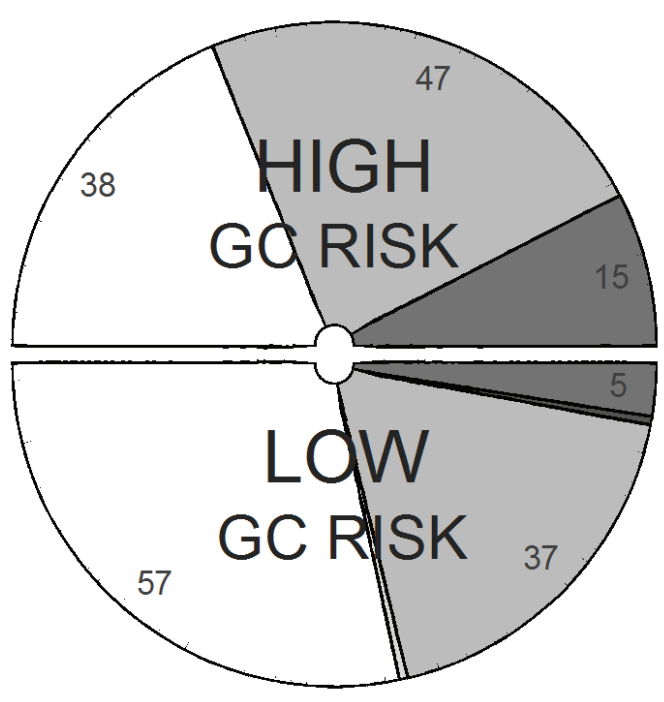

\section{Post-GC}

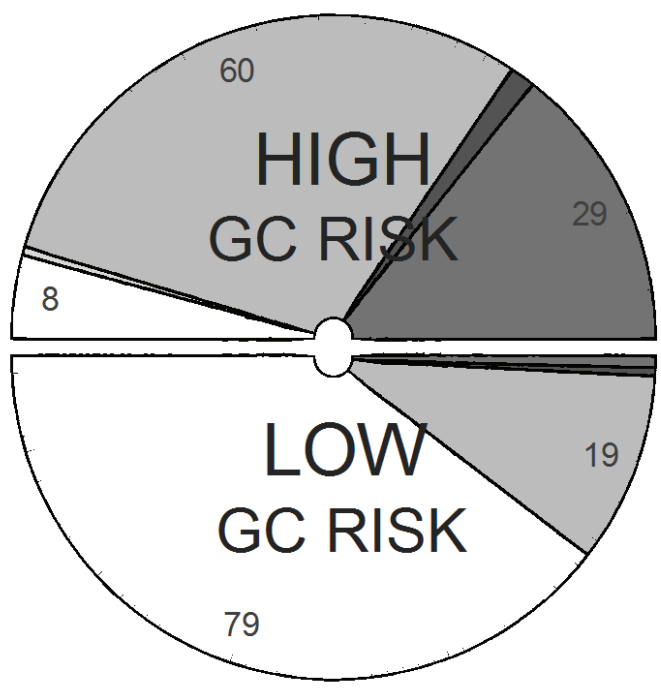

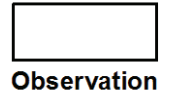

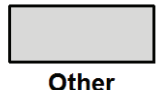

Other
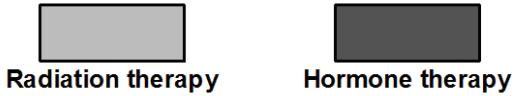

Radiation + hormone therapy

Figure 1: Breakdown of treatment recommendations pre-GC and post-GC for low and high GC risk groups in the adjuvant setting. Pre-GC treatment recommendations shown on left, post-GC treatment recommendation shown on right. Numbers indicate the $\%$ of patient cases for which a specific treatment recommendation was made in the respective GC group (100\% for each GC risk group). 
recommendation was made, urologists' confidence in treatment recommendations increased by $25 \%$ and $23 \%$ after reviewing the GC test result in the adjuvant and salvage settings, respectively. Additionally, urologists reported that the GC test result influenced their treatment recommendation in $84 \%$ (adjuvant) and $87 \%$ (salvage) of case evaluations (Table S4).

\section{DISCUSSION}

This clinical utility study was designed to prospectively assess the effect of a genomic classifier (GC) test that predicts metastasis following RP on urologists' adjuvant and salvage treatment recommendations. The performance of the GC test was previously reported in a blinded, independent validation study of a population of 1,010 men at high risk of recurrence (based on adverse pathology) post-RP [14]. That study revealed that $60 \%$ of clinically high-risk patients would be reclassified as low-risk with a cumulative incidence of metastasis of only $2.4 \%$ at 5 years post-RP. Conversely, patients with the highest GC scores (19\% of the cohort) had nearly 10 times higher cumulative incidence of metastasis by 5 years. Findings from this current study demonstrate that knowledge of the GC test result frequently impacted urologists' treatment recommendations in both the adjuvant (43\%) and salvage settings (53\%). Furthermore, in the adjuvant setting we were able to show that for patients with low GC risk, while pre-GC urologists recommended treatment $43 \%$ of the time, post-GC they were recommended to observation $79 \%$ of the time (Figure 1). Taken together, the clinical validation and utility results imply that among the population of prostate cancer patients at high-risk of recurrence following RP, the majority of patients tested will be recommended to close observation post-GC thereby sparing them the morbidity and costs associated with secondary therapy post-RP.

Guidelines on evidence development for molecular tests drafted in the past 3-5 years have urged going beyond obtaining evidence on an assay's analytical and clinical validity, encouraging additional research on how a test influences clinical practice management [15], [18]. To date in this nascent field, the number of published studies is fairly limited, but growing. In a clinical study assessing a molecular assay for stage II colon cancer, Srivastava et al. found that physicians changed chemotherapy decisions in $45 \%$ of patients [19], which fully validated predictions from a simulation of changes in NCCN guideline-directed treatment [20]. One of the most studied areas of practice management change in molecular medicine has been risk prediction in breast cancer. In a comprehensive and systematic review of clinical validity and changes in clinical practice patterns, Hornberger et al. found 15 studies reporting on 5 different tests [21]. They found that chemotherapy recommendation changed between $<1 \%-13 \%$ as reported in 4 studies of an online clinical

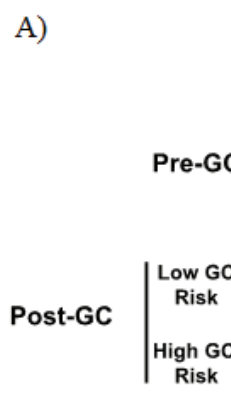

C)

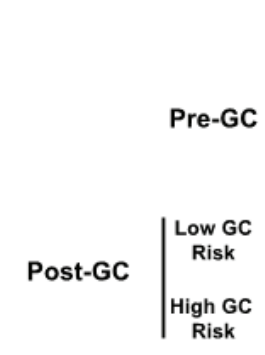

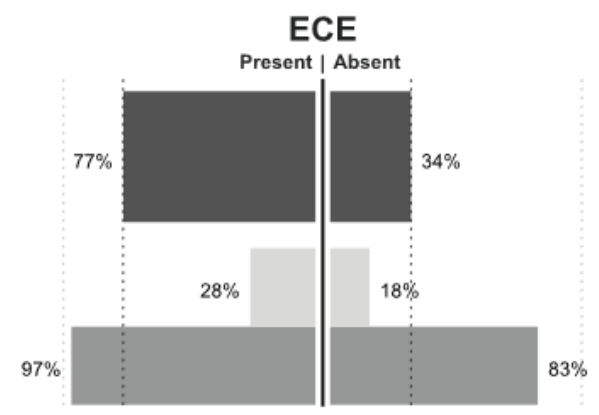

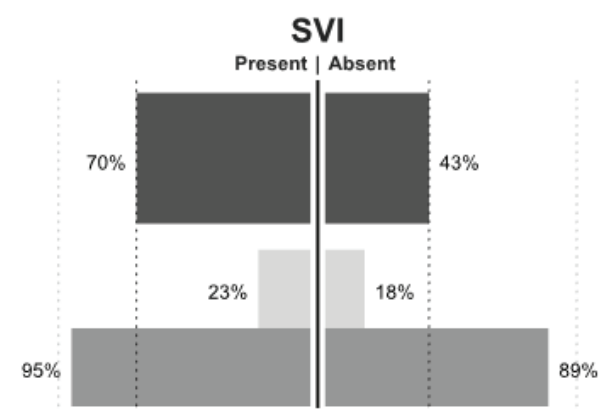

B)

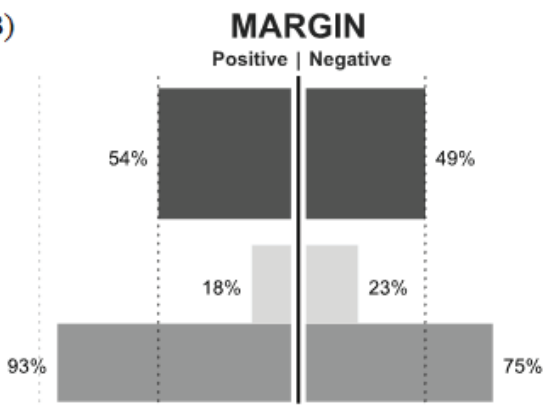

D)

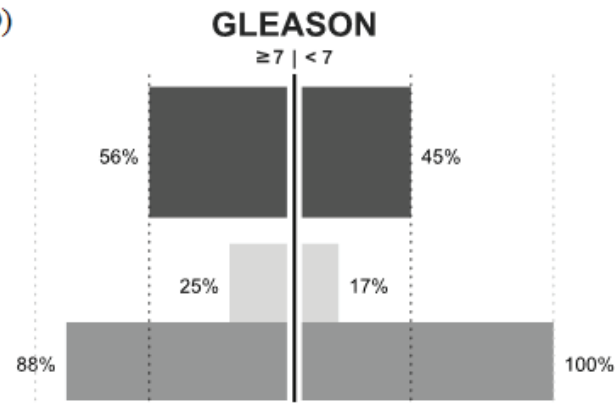

Figure 2: Proportion of recommendations for treatment for the indicated values of clinical variables (e.g. Presence/ Absence) Pre-GC and the resulting proportion recommended for treatment post-GC in high and low GC risk groups in the adjuvant setting. A) Extracapsular Extension (ECE), B) Surgical Margins, C) Seminal Vesicle Invasion (SVI) and D) Gleason score. Proportions of patients recommended treatment indicated on $\mathrm{x}$-axis (\%) depending on clinical variable status for pre-GC (dark grey, top), low GC risk (lightest grey, middle) and high GC risk (light grey, bottom). High GC tends to result in treatment rates higher than pre-GC while low GC tends to result in treatment rates lower than pre-GC. 


\begin{tabular}{|c|c|c|c|c|c|}
\hline Timepoint & Perceived Risk & GC Risk & Decrease & No Change & Increase \\
\hline \multirow[t]{4}{*}{ Adjuvant } & high & low & $20(50 \%)$ & $18(45 \%)$ & $2(5 \%)$ \\
\hline & & high & $3(5 \%)$ & $35(58.3 \%)$ & $22(36.7 \%)$ \\
\hline & low & low & $15(18.8 \%)$ & $60(75 \%)$ & $5(6.3 \%)$ \\
\hline & & high & $3(5 \%)$ & $24(40 \%)$ & $33(55 \%)$ \\
\hline \multirow[t]{3}{*}{ Salvage } & high & low & $48(45.7 \%)$ & $53(50.5 \%)$ & $4(3.8 \%)$ \\
\hline & & high & $1(3.3 \%)$ & $17(56.7 \%)$ & $12(40 \%)$ \\
\hline & low & high & $4(8.9 \%)$ & $15(33.3 \%)$ & $26(57.8 \%)$ \\
\hline \multicolumn{6}{|c|}{$\begin{array}{l}\text { Low (High) Perceived Risk }=<\text { half }(>\text { half }) \text { of clinicians initially recommend } \\
\text { treatment } \\
\text { Low (High) GC Risk at Advjuvant timepoint }=5 \text { year predicted probability }<6 \% \\
(>6 \%) \\
\text { Low (High) GC Risk at Salvage timepoint }=3 \text { year predicted probability }<18 \% \\
(>18 \%)\end{array}$} \\
\hline
\end{tabular}

decision support tool, compared with a median change across all studies of less than $35 \%$ in recommendations for a multi-gene assay. In comparison with these examples of accepted oncology tests, the finding in our study of a $43-53 \%$ change in recommendation upon receipt of the test results is supportive evidence that the GC test provides additional useful information to guide therapy selection.

This study reveals relevant findings relating to current practice patterns for high-risk prostate cancer patients post-RP and confirms urologists' proclivity for not only increased salvage treatment at the point of BCR but also increased intensification of treatment when compared to the adjuvant setting. Overall, urologists recommend treatment over 1.5 times as often in the salvage versus the adjuvant setting; treatment recommendations were made for $79 \%$ of case evaluations pre-GC in the salvage setting, 39\% of which involved a recommendation for multi-modal (i.e., radiation and hormone) therapy. This compares to a recommendation for multi-modal therapy in only $19 \%$ of case evaluations pre-GC in the adjuvant setting. In addition, the findings imply a potential to over-treat in the salvage setting as evidence suggests that even in patients presenting with BCR, less than one-third will go on to develop metastasis [5]. This is not without consequences for the patient and the healthcare system as both postoperative radiation and hormone therapy incur with considerable costs and morbidities including bladder and rectal complications as well as urinary incontinence and impotence, which can affect both near and long-term patient quality of life [22].

Results from this study also confirm that urologist decision-making in the adjuvant setting is mainly focused on whether or not to recommend postoperative radiation therapy. Prior to presentation of the GC test results, urologists recommend treatment in $52 \%$ of case evaluations with $99 \%$ of those recommendations including radiation therapy and only $20 \%$ of recommendations including hormone therapy. Accurate direction of radiation therapy to patients who are at highest biological risk for developing metastasis is critical as the morbidities and costs associated with treating patients with radiation modalities such as IMRT run high [23]. Furthermore, we observed that the GC risk significantly influenced the treatment recommendations irrespective of the presence or absence of specific clinical variables. Additionally, these findings confirm the widely accepted observation that in the salvage setting, the sensitivity of PSA rise may motivate urologists to recommend treatment despite its poor specificity. This hints towards a role for the GC test to improve urologist decision-making in this setting. Similar results were found relating to the intensification of treatment, where changes in intensity were driven primarily by GC risk rather than the perceived risk. This suggests that given the information from the GC test, presumably measuring the true biological potential of a patient's tumor, urologists are more willing to commit to the intensification of therapy than if this recommendation were solely based on rising PSA and clinical variables (i.e., pre-GC).

The results of this study provide insights to the potential role of the GC test despite the limitations of the study. Paramount among these is the non-random selection of cases and the fact that they do not represent the expected distribution of perceived or GC risk in the population at large. In particular, perceived and GC high-risk patients are over-represented in this study. Low GC risk cases tended to result in less treatment, but a larger study to more accurately estimate the influence in this substantial element of the population is necessary. Furthermore, the small number of physicians involved 
may not represent urologists at large; although accounting for intra-physician correlation (refer to Supplementary Material) resulted in a negligible impact on the overall probability of recommendation change. Additional studies are planned to include a larger random sample of both patient cases and urologists.

To our knowledge this is the first study to assess the effect of a molecular/genomic test on physician treatment recommendations in prostate cancer. Treatment recommendations changed in $43 \%$ of adjuvant setting case evaluations and $53 \%$ of salvage setting case evaluations. These findings demonstrate that knowledge of the genomic biomarker information in this GC test frequently influences these urologists' judgments about appropriate secondary therapy in both the adjuvant and salvage settings. These estimates are encouraging, but exclude near- and long-term implications on improved patient quality of life through more targeted recommendation of therapies, change in adverse event rates with secondary therapies such as radiation therapy, or direct costs associated with these therapies. Additionally, cost implications of supportive care, management of adverse events, delaying progression, or end-of-life care may provide a more comprehensive view of the true impact of novel genomic tests. As recommended in guidelines, it will be important to combine the findings of this study with other data to assess the E (i.e. ethical, legal and social implications) in the ACCE criteria.

\section{MATERIALS AND METHODS}

This clinical utility study used a prospective, prepost design, consisting of two independent sub-studies to assess patient cases at different points in patient management; both are collectively referred to herein as the DECIDE study. In one sub-study, urologists' treatment recommendations were assessed in the adjuvant setting, following RP without any evidence of PSA rise/BCR. In the other, treatment recommendations were assessed for a different cohort of cases in the salvage setting, following RP with evidence of PSA rise/BCR. Urologists were invited to review de-identified clinical variables on a set of twelve real patient cases in each sub-study and provide treatment recommendations. Cases were obtained from a previous clinical validation study $[13,14]$. In both the adjuvant and salvage sub-studies, recommendations were first obtained based solely on the clinical variables provided (pre-GC). Then, results of the GC test were assessed for the same de-identified cases and urologists were asked again to provide treatment recommendations (post-GC). Twenty urologists participated in the adjuvant setting study and 15 in the salvage setting study (Table 1).

The study was conducted in accordance with the Declaration of Helsinki and the Belmont report and was reviewed and approved by an independent IRB (Quorum Review Inc., Seattle, WA).
The primary objective of this study is to assess the effect of the GC test on urologists' adjuvant and salvage treatment recommendations for clinically and pathologically high-risk post-RP cases. Secondary objectives were to investigate specific changes in recommendations such as, proclivity of the GC test to result in more or less intensification of treatment, the relative importance of the $\mathrm{GC}$ to clinical variables and impact of the GC on urologists' confidence with treatment recommendations. Protocol-defined eligibility criteria for participation in the study required US board certified urologists practicing for at least 3 years and performing a high volume of RPs annually (Table 1). All urologists participating in the study were fellowship trained urologic oncologists. Eligible participants were identified through conference delegate lists and through established networks of key opinion leaders. Email invites were sent to 50 urologists meeting the inclusion criteria. Enrollment packages were sent to eligible urologists interested in participating in the study and included a cover letter, an educational primer on the GC test, a confidentiality agreement and a web link to the study's informed consent form (ICF) and electronic case report questionnaires (eCRQ).

Twenty-four high-risk, post-RP patient cases (12 adjuvant and 12 salvage) were selected for urologist review from the previously conducted clinical validation study $[13,14]$. The number of patient cases was selected to provide enough cases to adequately evaluate urologists' decision making across a range of high-risk patient types and was limited to twelve cases in each treatment setting so as to minimize study participant fatigue in reviewing cases. All cases were high-risk, post-RP as defined by the presence of one or more adverse pathological features including (1) pathological Gleason score 8+ or Gleason score 7 with primary pattern 4 ; (2) pathological stage T3a (ECE) or T3b (SVI); (3) positive surgical margins; or (4) Gleason grade upgrade from biopsy to RP. Selected cases did experience PSA nadir after RP.

These high-risk cases were further selected for inclusion in the study on the basis of the GC predicted probability of developing metastatic disease at 5 years post-RP and 3 years post-BCR for the adjuvant and salvage treatment settings, respectively. High (low) GC risk was defined as a 5- or 3-year predicted probability of metastasis greater (less) than $6 \%$ for the adjuvant setting and greater (less) than $18 \%$ for the salvage setting. In the adjuvant setting, six cases with low GC risk and six cases with high GC risk were selected. In the salvage setting, these numbers were 5 and 7 , respectively. Clinical assessment for each patient case was presented based on the following variables: age at surgery, pre-operative PSA levels, pathologic stage, biopsy and pathologic Gleason score, presence or absence of SVI and ECE, surgical margin status and lymph node involvement (Table 2). Additionally, PSAdt and time to BCR were provided for 
cases evaluated in the salvage setting. These thresholds were based on the average risk of metastasis in the original study population $[13,17]$. Actual patient outcomes (i.e.: progression status at any time point) were not presented for assessment.

All cases were de-identified and presented in a randomized fashion to eliminate bias toward the urologist's pre- and post-GC treatment recommendations. Cases were randomized both from urologist to urologist and from pre- to post-GC. Clinical variables and GC test result information were provided to urologists through a secure online platform, and all treatment recommendations were collected using the eCRQ. Treatment recommendations included referral to a radiation oncologist and/or initiation of hormones, close observation, or any other recommendation not listed on the eCRQ.

Confidence intervals for probability of recommendation change from pre- to post-GC were constructed using a normal approximation, a significance level of $5 \%$, and all recommendations were considered as independent. Chi-squared tests were used for univariate assessment of treatment predictors and multivariable analyses were performed using logistic regression. All statistical analyses were performed using SAS 9.2 (Cary, NC). All tests were 2-sided with a Type I error probability of $5 \%$.

\section{CONCLUSION}

The DECIDE study assessed the effect of the GC test on urologist treatment recommendations for high-risk case evaluations in the adjuvant and salvage treatment settings post-RP. Findings demonstrate that knowledge of the GC test result frequently impacted urologists' treatment recommendations in both the adjuvant and salvage settings. Furthermore, the GC test appears to better direct urologist treatment recommendations irrespective of the presence or absence of conventional pathology and clinical variables that are currently used to assess risk in these patients.

In conclusion, this study suggests that when implemented into routine clinical practice, the GC test has the potential to change treatment recommendations after radical prostatectomy and better identify patients that may benefit from intensive multimodal therapy, while sparing those who can be closely observed without initiating aggressive secondary therapy and in doing so, holds the potential to improve patient outcomes, decrease morbidities and ultimately reduce costs to the healthcare system.

\section{DISCLOSURE / CONFLICT OF INTEREST}

This study was supported by GenomeDx Biosciences. 4 co-authors are employees of the sponsor.

\section{ACKNOWLEDGEMENTS}

The authors thank Drs. R. Jeffrey Karnes and Robert B. Jenkins (Mayo Clinic, Rochester MN) for access to the Mayo Clinic Prostate Cancer Radical Prostatectomy Registry, Dr. Leslie Esford (National Research Council of Canada Industrial Research Assistance Program) for funding, Dr. Ismael A. Vergara, Mark Friedman and Harlan Campbell for helpful comments and assistance in preparing this manuscript.

\section{REFERENCES}

1. Siegel R, Naishadham D and Jemal A. Cancer statistics, 2013. CA: a cancer journal for clinicians. 2013; 63(1):1130.

2. Marciscano AE, Hardee ME and Sanfilippo N. Management of high-risk localized prostate cancer. Advances in urology. 2012; 2012:641689.

3. Swanson GP, Riggs M and Hermans M. Pathologic findings at radical prostatectomy: risk factors for failure and death. Urologic oncology. 2007; 25(2):110-114.

4. NCCN. NCCN Clinical Guidelines in Oncology (NCCN Guideline). Prostate Cancer. Version 1. 2013.

5. Pound CR, Partin AW, Eisenberger MA, Chan DW, Pearson JD and Walsh PC. Natural history of progression after PSA elevation following radical prostatectomy. JAMA: the journal of the American Medical Association. 1999; 281(17):1591-1597.

6. Boorjian SA, Thompson RH, Tollefson MK, Rangel LJ, Bergstralh EJ, Blute ML and Karnes RJ. Long-term risk of clinical progression after biochemical recurrence following radical prostatectomy: the impact of time from surgery to recurrence. European urology. 2011; 59(6):893-899.

7. Freedland SJ, Humphreys EB, Mangold LA, Eisenberger M, Dorey FJ, Walsh PC and Partin AW. Death in patients with recurrent prostate cancer after radical prostatectomy: prostate-specific antigen doubling time subgroups and their associated contributions to all-cause mortality. Journal of clinical oncology : official journal of the American Society of Clinical Oncology. 2007; 25(13):1765-1771.

8. Bolla M, van Poppel H, Collette L, van Cangh P, Vekemans K, Da Pozzo L, de Reijke TM, Verbaeys A, Bosset J-F, van Velthoven R, Maréchal J-M, Scalliet P, Haustermans $\mathrm{K}$ and Piérart M. Postoperative radiotherapy after radical prostatectomy: a randomised controlled trial (EORTC trial 22911). The Lancet. 2005; 366(9485):572-578.

9. Thompson IM, Tangen CM, Paradelo J, Lucia MS, Miller G, Troyer D, Messing E, Forman J, Chin J, Swanson G, Canby-Hagino E and Crawford ED. Adjuvant radiotherapy for pathological T3N0M0 prostate cancer significantly reduces risk of metastases and improves survival: longterm followup of a randomized clinical trial. The Journal of urology. 2009; 181(3):956-962. 
10. Wiegel T, Bottke D, Steiner U, Siegmann A, Golz R, Storkel S, Willich N, Semjonow A, Souchon R, Stockle M, Rube C, Weissbach L, Althaus P, Rebmann U, Kalble T, Feldmann HJ, et al. Phase III postoperative adjuvant radiotherapy after radical prostatectomy compared with radical prostatectomy alone in pT3 prostate cancer with postoperative undetectable prostate-specific antigen: ARO 96-02/AUO AP 09/95. Journal of clinical oncology : official journal of the American Society of Clinical Oncology. 2009; 27(18):2924-2930.

11. Lughezzani G, Briganti A, Karakiewicz PI, Kattan MW, Montorsi F, Shariat SF and Vickers AJ. Predictive and prognostic models in radical prostatectomy candidates: a critical analysis of the literature. European urology. 2010; 58(5):687-700.

12. Erho N, Crisan A, Vergara IA, Mitra AP, Ghadessi M, Buerki C, Bergstralh EJ, Kollmeyer TM, Fink S, Haddad Z, Zimmermann B, Sierocinski T, Ballman KV, Triche TJ, Black PC, Karnes RJ, et al. Discovery and Validation of a Prostate Cancer Genomic Classifier that Predicts Early Metastasis Following Radical Prostatectomy. PLoS One. 2013; Accepted

13. Ghadessi M, Bergstralh EJ, Erho N, Crisan A, Davicioni E, Buerki C, Mitra AP, Thompson DJS, Carlson R, Haddad Z, Zimmermann B, Ballman KV, Kollmeyer TM, Sierocinski $\mathrm{T}$, Vergara IA, Triche TJ, et al. Validation of a genomic classifier that predicts metastatic disease progression in men with high-risk pathologic features postprostatectomy. Journal of Clinical Oncology. 2013; 31(suppl 6; abstr 36).

14. Davicioni E, Ghadessi M, Bergstralh EJ, Buerki C, Mitra AP, Crisan A, Erho N, Vergara IA, Carlson R, Thompson DJS, Haddad Z, Zimmermann B, Sierocinski T, Triche TJ, Fink S, Kollmeyer TM, et al. Validation of a genomic classifier that predicts metastasis following radical prostatectomy in an at risk patient population. In submission. 2013.

15. Hornberger J, Doberne $\mathrm{J}$ and Chien R. Laboratorydeveloped test--SynFRAME: an approach for assessing laboratory-developed tests synthesized from prior appraisal frameworks. Genetic testing and molecular biomarkers. 2012; 16(6):605-614.

16. Genetic Testing. ACCE model system for collecting, analyzing and disseminating information on genetic tests. 2007. CDC.

17. Ross AE, Ghadessi M, Davicioni E, Crisan A, Buerki C, Erho N, Mitra AP, Thompson DJS, Triche TJ, Schaeffer EM and Feng FYC. Validation of a genomic classifier that predicts metastatic disease progression in men with biochemical recurrence post radical prostatectomy. Journal of Clinical Oncology. 2013; 31(suppl 6; abstr 52).

18. Teutsch SM, Bradley LA, Palomaki GE, Haddow JE, Piper M, Calonge N, Dotson WD, Douglas MP and Berg AO. The Evaluation of Genomic Applications in Practice and Prevention (EGAPP) Initiative: methods of the EGAPP Working Group. Genetics in medicine : official journal of the American College of Medical Genetics. 2009; 11(1):314.

19. Srivastava G, Renfro LA, Behrens RJ, Lopatin M, Chao C, Soori GS, Dakhil SR, Mowat RB, Kuebler JP, Kim GP, Mazurczak M, Lee M and Alberts SR. Prospective evaluation of a 12-gene assay on treatment recommendations in patients with stage II colon cancer. Journal of Clinical Oncology. 2012; 30 (suppl 34, abstr 453).

20. Hornberger J, Lyman GH, Chien R and Meropol NJ. A Multigene Prognostic Assay for Selection of Adjuvant Chemotherapy in Patients with T3, Stage II Colon Cancer: Impact on Quality-Adjusted Life Expectancy and Costs. Value in health : the journal of the International Society for Pharmacoeconomics and Outcomes Research. 2012; 15(8):1014-1021.

21. Hornberger J, Alvarado MD, Rebecca C, Gutierrez HR, Yu TM and Gradishar WJ. Clinical validity/utility, change in practice patterns, and economic implications of risk stratifiers to predict outcomes for early-stage breast cancer: a systematic review. Journal of the National Cancer Institute. 2012; 104(14):1068-1079.

22. Krupski TL, Foley KA, Baser O, Long S, Macarios D and Litwin MS. Health care cost associated with prostate cancer, androgen deprivation therapy and bone complications. The Journal of urology. 2007; 178(4 Pt 1):1423-1428.

23. Hodges JC, Lotan Y, Boike TP, Benton R, Barrier A and Timmerman RD. Cost-Effectiveness Analysis of SBRT Versus IMRT: An Emerging Initial Radiation Treatment Option for Organ-Confined Prostate Cancer. American Journal of Managed Care. 2012; 18(5 Spec No.2):e186-e193. 